\title{
Serpin Peptidase Inhibitor Clade A Member 1 (SerpinA1) Is a Novel Biomarker for Progression of Cutaneous Squamous Cell Carcinoma
}

\author{
Mehdi Farshchian, ${ }^{\star \dagger}$ Atte Kivisaari, ${ }^{* \dagger}$ \\ Risto Ala-aho, ${ }^{\star \dagger}$ Pilvi Riihilä, ${ }^{\star \dagger}$ Markku Kallajoki, ${ }^{\ddagger}$ \\ Reidar Grénman, ${ }^{\S}$ Juha Peltonen," \\ Taina Pihlajaniemi," Ritva Heljasvaara," \\ and Veli-Matti Kähäri ${ }^{\star \dagger}$

\begin{abstract}
From the Departments of Dermatology," Pathology, ${ }^{\ddagger}$ and Otorhinolaryngology-Head and Neck Surgery, ${ }^{\S}$ University of Turku and Turku University Hospital, Turku; the MediCity Research Laboratory, ${ }^{\dagger}$ and the Department of Anatomy and Cell Biology, "Iniversity of Turku, Turku; and the Biocenter Oulu and the Department of Medical Biochemistry and Molecular
\end{abstract} \\ Biology," University of Oulu, Oulu, Finland
}

The incidence of keratinocyte-derived nonmelanoma skin cancers is increasing worldwide because of cumulative recreational exposure to sunlight. At present, no specific molecular markers are available for assessing the progression of premalignant actinic keratoses to invasive cutaneous squamous cell carcinoma (SCC). We examined the role of the Serpin family in skin SCCs. Expression profiling of cutaneous SCC cell lines $(n=8)$ revealed up-regulation of SerpinA1 compared with normal epidermal keratinocytes $(n=5)$. Analysis with quantitative RT-PCR showed that the mean level of SerpinA1 mRNA was markedly up-regulated in cutaneous SCC cell lines $(n=8)$ compared with in normal keratinocytes. SerpinA1 production by SCC cells was dependent on $\mathbf{p 3 8}$ mitogen-activated protein kinase activity and was upregulated by epidermal growth factor, tumor necrosis factor- $\alpha$, interferon- $\gamma$, and IL-1 $\beta$. Immunostaining of tissue arrays with 148 human tissue samples revealed tumor cell-associated expression of SerpinA1 in 19 of 36 actinic keratoses, 22 of 29 Bowen's disease samples, 67 of 71 sporadic SCCs, and all 12 recessive dystrophic epidermolysis bullosa-associated SCCs examined. Moreover, tumor cell-associated SerpinA1 staining was detected in all chemically induced mouse skin SCCs studied $(n=17)$. Overexpression of SerpinA1 mRNA was also detected by quantitative RT-PCR in chemically induced mouse skin SCCs $(n=14)$ compared with con- trol tissues $(n=14)$. These data identify SerpinA1 as a novel tumor cell-associated biomarker for progression of cutaneous SCCs. (AmJ Pathol 2011, 179:1110-1119; DOI: 10.1016/j.ajpath.2011.05.012)

The incidence of melanoma and nonmelanoma skin cancer is increasing globally. ${ }^{1-3}$ Nonmelanoma skin cancers, including basal cell carcinoma (approximately $80 \%$ ) and squamous cell carcinoma (SCC) (approximately $20 \%$ ), are among the most common cancers worldwide, and SCC has been reported as the second most common cutaneous malignancy in the white population. ${ }^{1-3}$ Although early excision of cutaneous SCC is associated with a favorable outcome, for patients with metastatic disease (6\%), the long-term prognosis is poor. ${ }^{4}$ Important risk factors for cutaneous SCC include exposure to UV radiation, immunosuppression, and chronic skin ulceration. ${ }^{1-3}$ An example of the latter is individuals with recessive dystrophic epidermolysis bullosa (RDEB), who often develop rapidly progressing cutaneous SCCs at sites of chronic ulceration and scarring. ${ }^{5,6}$ At present, no specific molecular markers for progression of cutaneous SCC are available. Such biomarkers would be valuable in clinical practice for early

Supported by grants from the Academy of Finland (projects 114409 , 130224, and 137687), Turku University Hospital (projects 13336 and 13010), the Dystrophic Epidermolysis Bullosa Research Association, the Sigrid Jusélius Foundation, the Cancer Research Foundation of Finland, The Southwestern Finland Cancer Societies, and personal grants from the Centre for International Mobility, the Turku University Foundation, and the Finnish Cultural Foundation (M.F.) and from the Finnish Dermatological Society, the Finnish Society of Dermatopathology, and the K. Albin Johansson Foundation (A.K.).

Accepted for publication May 4, 2011.

A.K. and P.R. are doctoral students in the National Graduate School of Clinical Investigation (CLIGS).

M.F. and A.K. contributed equally to this work.

Address reprint requests to Veli-Matti Kähäri, M.D., Ph.D., Department of Dermatology, University of Turku and Turku University Hospital, PO Box 52, Fl-20521 Turku, Finland. E-mail: veli-matti.kahari@utu.fi. 
detection of individual cutaneous SCCs with a high risk of progression and metastasis.

Serine protease inhibitors (serpins) constitute the largest and most broadly distributed superfamily of protease inhibitors described in humans, with the two largest clades of the 36 serpins consisting of extracellular molecules "clade A" and intracellular serpins "clade B." Serpin peptidase inhibitor clade A member 1 (SerpinA1), also known as $\alpha_{1}$-proteinase inhibitor or $\alpha_{1}$-antitrypsin (AAT), is a highly effective inhibitor of neutrophil elastase, which also inhibits the activity of plasmin, thrombin, trypsin, chymotrypsin, and plasminogen activator. ${ }^{7,8}$ Patients with AAT deficiency carry an increased risk of emphysema and liver disease. ${ }^{9}$ Another member of the serpin superfamily with medical importance is serpin peptidase inhibitor clade A member 3 (SerpinA3), also known as $\alpha_{1}$-antichymotrypsin (ACT), which displays inhibitory function toward neutrophil cathepsin $\mathrm{G}$ and mast cell chymase and serves as an inflammatory response molecule and an acute-phase reactant protein. ${ }^{7,8}$ Structural variants of ACT protein have been implicated in Alzheimer's disease, ${ }^{10,11}$ and combined deficiency of AAT and ACT increases the risk of chronic liver disease. ${ }^{12}$ Elevated expression of AAT is associated with the invasive and metastatic potential and poor prognosis in lung, colorectal, and gastric carcinoma. ${ }^{13-16}$ In addition, ACT is expressed at high levels in gastric and salivary gland cancer and in malignant melanoma. ${ }^{16-18}$

Herein, we examined Serpin expression in cutaneous SCCs. The results show that SerpinA1 is expressed by cutaneous SCC cells in culture and by tumor cells in SCCs of the skin. The level of expression is low in premalignant lesions of skin (actinic keratoses) and is clearly elevated in invasive cutaneous SCCs. In addition, tumor cell-associated SerpinA1 staining was detected in mouse skin SCCs. These results identify SerpinA1 as a novel tumor cell-associated diagnostic biomarker for progression of cutaneous SCC.

\section{Materials and Methods}

\section{Ethical Issues}

The use of archival tissue specimens and the collection of normal skin and SCC tissues was approved by the Ethics Committee of the Hospital District of Southwest Finland, Turku, Finland. Before surgery, each patient gave their informed consent, and the study was conducted according to the Declaration of Helsinki.

The animal experiments were approved by the State Provincial Office of Southern Finland.

\section{Cell Cultures}

Human cutaneous SCC cell lines $(n=8)$ were established from surgically removed SCCs of skin. ${ }^{19-21}$ SCC cells were cultured in Dulbecco's modified Eagle's medium supplemented with $6 \mathrm{mmol} / \mathrm{L}$ glutamine, nonessential amino acids, and $10 \%$ fetal calf serum. ${ }^{20,21} \mathrm{HaCaT}$, a spontaneously immortalized, nontumorigenic human epidermal keratinocyte-derived cell line, ${ }^{22}$ and the Ha-ras- transformed tumorigenic HaCaT cell lines A5, II4, and RT3 ${ }^{23,24}$ (a gift from Dr. Norbert E. Fusenig, German Cancer Research Center, Heidelberg, Germany) were cultured in Dulbecco's modified Eagle's medium containing $10 \%$ fetal calf serum. G418 (200 $\mu \mathrm{g} / \mathrm{mL})$ was added to the medium of the Ha-ras-transformed HaCaT cell lines. ${ }^{23,24}$ Normal human epidermal keratinocytes (NHEKs) were cultured from nonmalignant skin of patients $(n=4)$ undergoing surgery for mammoplasty at Turku University Hospital, Turku, Finland. In addition, normal keratinocytes were purchased from PromoCell (Heidelberg, Germany). Keratinocytes were cultured in keratinocyte basal medium 2, supplemented with SingleQuots (Cambrex Bioscience, Walkersville, MD), as previously described. ${ }^{20,21}$

\section{Human Tissue Samples}

Tissue collection was performed at the Department of Pathology, Turku University Hospital. ${ }^{6,25}$

\section{Mouse Skin Chemical Carcinogenesis}

Normal $(n=5)$, acetone-treated $(n=2)$, and hyperplastic skin $(n=6)$ and SCC $(n=17)$ samples were collected from skin of FVB/N HanHsd mice (maintained at the Laboratory Animal Centre, University of Oulu, Oulu, Finland). Mouse skin carcinogenesis was induced as previously described. ${ }^{26}$ Briefly, to induce hyperplasia, the shaved dorsal skin was treated four times, at 2-day intervals, with $5 \mu \mathrm{g}$ of 12-O-tetradecanoylphorbol-13-acetate (TPA; Sigma-Aldrich, St Louis, MO,) in $100 \mu \mathrm{L}$ of acetone. The control mice were treated in a similar way four times with acetone. To induce skin tumors, a single dose of $100 \mu \mathrm{g}$ of 7,12-dimethylbenz [ $\alpha$ ]anthracene (DMBA, Sigma-Aldrich) in $100 \mu \mathrm{L}$ of acetone was administered topically on the shaved dorsal skin, followed by weekly TPA treatments (5 $\mu \mathrm{g}$ in $100 \mu \mathrm{L}$ of acetone) for 20 weeks. Mice were monitored weekly for the appearance of tumors and were sacrificed at the end of the experiment at week 32 or earlier when the tumor load was excessive, invasive carcinomas appeared, or the diameter of an individual tumor exceeded $10 \mathrm{~mm}$. Approximately $10 \%$ of the benign papillomas progress to malignant SCCs in this model. Tumors and skin samples were removed and fixed in fresh phosphate-buffered $4 \%$ paraformaldehyde for 24 hours at $4^{\circ} \mathrm{C}$ and were embedded in paraffin. The TPA-treated skin and SCC samples were evaluated on the basis of H\&E-stained sections.

\section{RNA Extraction and cDNA Preparation}

Twenty-four hours before the RNA extraction, the culture media of cells was replaced by serum-free culture media. Total RNA was isolated from cultured cells and tissue samples using the RNeasy kit (Qiagen, Chatworth, CA) according to the manufacturer's protocol. cDNA was reverse transcribed from $1 \mu \mathrm{g}$ of RNA using reverse transcriptase M-MLV RNase $\mathrm{H}$ without reverse transcriptase and random hexamers (Promega, Helsinki, Finland). ${ }^{20,21}$ 


\section{Affymetrix Microarray Analysis}

The gene expression profiling of cutaneous SCC cell lines $(n=8)$ and NHEKs from healthy individuals $(n=5)$ for the entire serpin family was performed using Affymetrix Human U133 Plus 2.0 GeneChips (Affymetrix Inc., Santa Clara, CA) at the Finnish Microarray and Sequencing Centre, Turku Center for Biotechnology. Normalization of the arrays was performed using robust multiarray average assay, Chipster software (CSC, Espoo, Finland). The mean signal level of NHEKs was used as the control for the signal level of each cell line. The sequence specificity of Affymetrix probes was verified by Basic Local Alignment Search Tool search.

\section{Quantitative Real-Time PCR}

The primers and probes were designed based on human sequences for the genes tested and purchased from Oligomer (Helsinki, Finland): SerpinA1 forward primer, 5'-CACCGTGAAGGTGCCTATGATG-3'; SerpinA1 reverse primer, 5'-GGCATTGCCCAGGTATTTCATC-3'; SerpinA1 probe, 5'-Fam-TAGGCATGTTTAACATCCAGCACTGTA-Tamra-3'; SerpinA3 forward primer, 5' -GCATCACCTGACTATACCT-3'; SerpinA3 reverse primer, 5'-GCATTGCCTGTGTACTTC-3'; and SerpinA3 probe, 5'Fam-TACTTCCGGGACGAGGAGCTGTC-BHQ1-3'. One set of primers and probe were designed for murine SerpinA1 mRNAs based on homologous sequence in the coding regions of five murine SerpinA1 genes (a-e): murine SerpinA1 forward primer, 5'-CCTCAACAGACCAGACAGTGA-3'; murine SerpinA1 reverse primer, 5'-CTCCACCAGCTTCAGGTCAT-3'; and murine SerpinA1 probe, 5'Fam-CTGCAGCTGAGCACAGGCAATGG-BHQ1-3'.

RT-PCR was performed using an ABI 7700 sequence detector (Applied Biosystems, Warrington, UK) as outlined previously. ${ }^{20,21}$ The levels of $\beta$-actin and glyceraldehyde-3phosphate dehydrogenase mRNA (as housekeeping genes) were determined as described previously. ${ }^{21}$

\section{Western Blot Analysis}

SerpinA1 production by cutaneous SCC cell lines and NHEKs was analyzed by Western blotting of conditioned culture media after fractionation by $10 \%$ SDS-PAGE ${ }^{27}$ using polyclonal rabbit anti-human AAT (SerpinA1; DakoCytomation, Glostrup, Denmark). Equal protein loading was ensured by Western blot analysis of corresponding cell lysates with an antibody against $\beta$-actin (A1978; Sigma-Aldrich).

\section{Regulation of SerpinA1 Expression by SCC Cells}

To investigate the regulation of SerpinA1 expression with inflammatory cytokines and growth factors, the cutaneous SCC cell line (UT-SCC-118) was cultured and serum starved for 24 hours before treatment with epidermal growth factor (EGF; $25 \mathrm{ng} / \mathrm{mL}$ ) (Roche Diagnostics $\mathrm{GmbH}$, Mannheim, Germany), tumor necrosis factor- $\alpha$ (TNF- $\alpha ; 20 \mathrm{ng} / \mathrm{mL}$ ) (Sigma-Aldrich), interferon- $\gamma$ (IFN- $\gamma$; $100 \mathrm{U} / \mathrm{mL}$ ) (Promega), and $\mathrm{IL}-1 \beta$ (10 ng/mL) (Calbio- chem, San Diego, CA). Total RNA was isolated and cDNAs were prepared for quantitative real-time PCR as described previously herein. Western blot analysis of the conditioned media for SerpinA1 was performed as described previously herein. As loading control, cell lysates were analyzed for $\beta$-actin by Western blotting, as mentioned previously herein.

To study the role of mitogen-activated protein kinase (MAPK) signaling on the expression of SerpinA1, SCC cell cultures were serum starved for 24 hours and then treated with MEK1/2 inhibitor PD98059 (30 $\mu \mathrm{mol} / \mathrm{L}$ ) or with p38 MAPK inhibitor SB203580 (10 $\mu \mathrm{mol} / \mathrm{L})$ (both from Calbiochem) for 24 hours. SerpinA1 levels in conditioned medium were determined as described previously herein. The functions of the inhibitors PD98059 and SB203580 were verified by Western blot analysis of the cell lysates with antibodies specific for phospho-ERK1/2 and phospho-CREB (both from Cell Signaling Technology Inc., Beverly, MA), respectively. 25,27

\section{IHC Analysis}

The tissue used for immunohistochemical (IHC) staining consisted of archival formalin-fixed, paraffin-embedded (FFPE) tissue samples as follows: actinic keratosis ( $n=$ $36)$, Bowen's disease $(n=29)$, sporadic UV-induced cutaneous SCC $(n=71)$, and RDEB-associated cutaneous SCC $(n=12){ }^{6,25}$ To enhance the comparability and reliability of the $\mathrm{IHC}$ analysis results, we used an automated immunostaining device and the tissue microarray (TMA) technique. TMA blocks were generated from the original paraffin blocks using a tissue arrayer (Beecher Instruments Inc., Sun Prairie, WI), as previously described. ${ }^{28}$ Immunolabeling was performed on the $5-\mu \mathrm{m}$ thick TMA sections using an automated immunostaining device (Ventana Medical Systems SA, Illkirch, France) for analysis of the human tissue samples. Mouse skin SCC tumors and mouse control tissue samples were analyzed as whole sections. We used, as a primary antibody, polyclonal rabbit anti-human SerpinA1 antibody, which crossreacts with rodent SerpinA1, 1:800 (ATT, code No. A0012; DakoCytomation). The primary antibody was detected using the Ventana ultraView Universal DAB detection kit and the Ventana amplification kit (Ventana Medical Systems SA). All the samples were examined independently by light microscopy by two observers (M.F. and A.K.). For semiquantitative evaluation of SerpinA1 immunostainings, membranous and cytoplasmic SerpinA1 stainings in tumor cells were considered positive. SerpinA1 immunostaining was scored semiquantitatively as follows: negative $(-)$, weak $(+)$, moderate $(++)$, and strong $(+++)$. For negative control stainings, the primary antibody was omitted and replaced by PBS.

\section{Statistical Analysis}

To evaluate the gene expression differences between the groups, we used Student's t-test. Statistical analysis of the immunostaining results was performed by comparing the differences in immunoreactivity between different tissue types using the nonparametrical Pearson $\chi^{2}$ test. For 

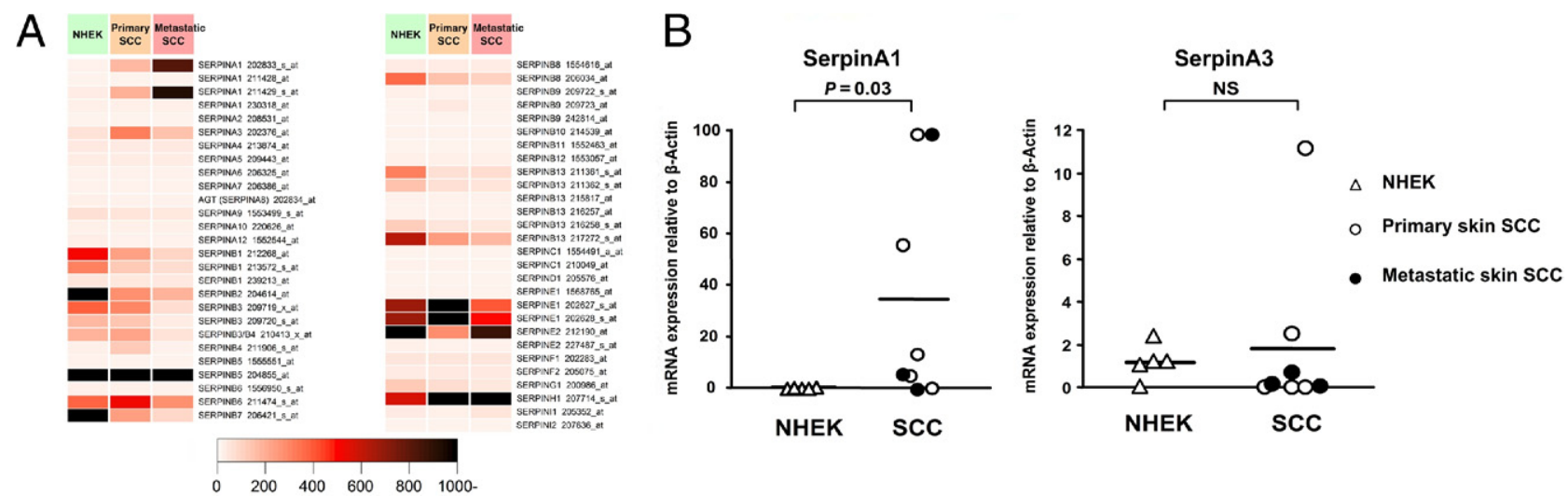

Figure 1. Overexpression of SerpinA1 in cutaneous SCC cell lines. A: Oligonucleotide array (Affymetrix)-based analysis of Serpin expression in primary ( $n=$ 5) and metastatic $(n=3)$ human cutaneous SCC cell lines and in primary NHEKs from different individuals $(n=5)$. B: SerpinA1 and SerpinA3 mRNA levels in SCC cell lines and normal keratinocytes, as in $\mathbf{A}$, were quantified by real-time PCR, and the levels were corrected for $\beta$-actin mRNA levels in the same samples. Horizontal bars represent the mean SerpinA1 expression level for each group. Statistical significance was determined by Student's $t$-test. NS, not significant.

statistical analysis, semiquantitative immunostaining results were regrouped in a way that negative $(-)$ and weak $(+)$ staining results were considered as one group and moderate $(++)$ and strong $(+++)$ semiquantitative results were considered as the other group.

\section{Results}

\section{SerpinA1 Expression Is Up-Regulated in Cutaneous SCC Cells}

Expression profiling of the entire Serpin gene family in primary $(n=5)$ and metastatic $(n=3)$ cutaneous SCC cell lines and primary NHEKs from healthy individuals $(n=5)$ was performed using oligonucleotide-based microarrays. The analysis revealed expression of several distinct Serpin gene family members in NHEKs (Figure 1A). In addition, several serpin family members (SerpinA1, SerpinA3, SerpinB5, SerpinB6, SerpinE1, SerpinE2, and SerpinH1) were also expressed at high levels in primary and metastatic skin SCC cells. Comparison of the Serpin gene expression levels between epidermal keratinocytes and cutaneous SCC cell lines revealed elevated expression of SerpinA1 and SerpinA3 in SCC cells compared with that in normal keratinocytes (Figure 1A).

To verify the microarray-based results, the expression of SerpinA1 and SerpinA3 mRNAs in cutaneous SCC cells and NHEKs was analyzed by quantitative real-time PCR and was corrected for $\beta$-actin mRNA levels in the same samples. The results revealed marked and significant (50-fold; $P=0.03$ ) up-regulation of SerpinA1 mRNA in cutaneous SCC cell lines compared with that in NHEKs (Figure 1B). In contrast, most cutaneous SCC cell lines showed low expression of SerpinA3 mRNA, whereas notable expression of SerpinA3 was observed in NHEKs (Figure 1B). No significant difference in the levels of SerpinA3 mRNA were detected between cutaneous SCC cells and NHEKs $(P=0.66)$.

\section{SerpinA1 Production Is Up-Regulated in Cutaneous SCC Cells}

Production of SerpinA1 protein by cutaneous SCC cells and epidermal keratinocytes was also examined by Western blot analysis of conditioned media of the cultures. Presence of the specific 51-kDa band corresponding to SerpinA1 was noted in aliquots of conditioned media of all cutaneous SCC cell lines (Figure 2A). In contrast, only one of five NHEK cell lines produced detectable levels of SerpinA1 (Figure 2A).

\section{Expression of SerpinA1 Correlates with Tumorigenic Potential of Transformed Keratinocytes}

The previous results suggest that the expression of SerpinA1 in cutaneous SCC cells is induced as a result of malignant transformation of epidermal keratinocytes. To further investigate this hypothesis, we examined the expression of SerpinA1 mRNA in an immortalized nontumorigenic cell line $(\mathrm{HaCaT})$ derived from human epidermal keratinocytes and in three Ha-ras-transformed $\mathrm{HaCaT}$ cell lines (A5, II4, and RT3), which represent an in vitro model for different stages of SCC tumor progression. Specifically, A5 is a benign tumorigenic HaCaT-derived cell line, II4 forms invasive malignant tumors, and RT3 cells form metastatic tumors in vivo. ${ }^{23,24}$ Nontumorigenic $\mathrm{HaCaT}$ cells, with inactivation of both alleles of $p 53$ tumor suppressor, express low levels of SerpinA1 mRNA (Figure 2B). In contrast, significantly higher expression of SerpinA1 mRNA was noted in Ha-ras-transformed $\mathrm{HaCaT}$ cell lines, and the expression correlated with tumorigenic potential because it was lowest in the A5 cell line and highest in the RT3 cell line (Figure 2B). These results suggest that SerpinA1 serves as a marker for the malignant transformation of NHEKs to the aggressive and metastatic forms of SCC. 
A
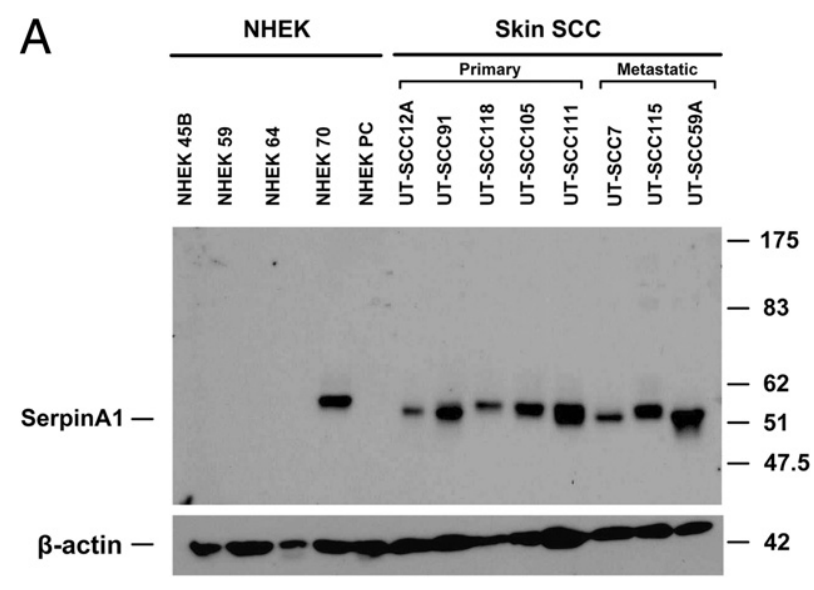

B

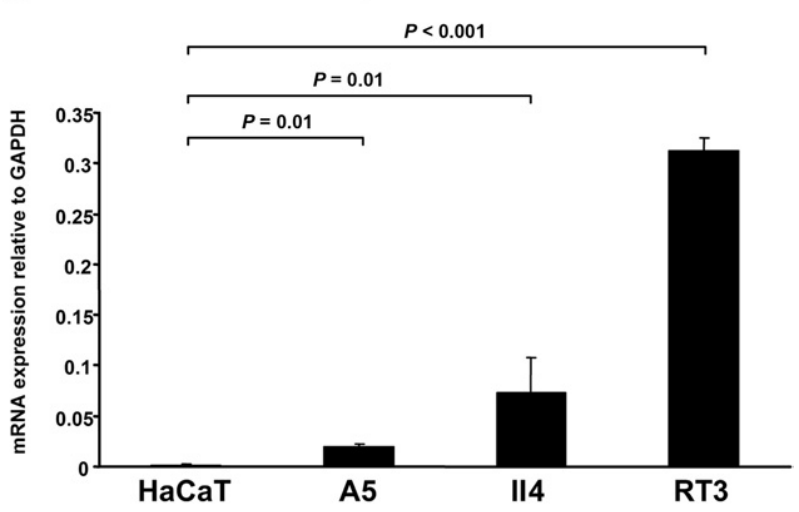

Figure 2. SerpinA1 overexpression in cutaneous SCC cells and tumorigenic ras-transformed HaCaT cell lines in culture. A: SerpinA1 levels in conditioned media of NHEKs and cutaneous SCC cell lines were determined by Western blot analysis. $\beta$-Actin levels were determined in the corresponding cell lysates as the loading control. Migration positions of molecular weight markers (in kilodaltons) are shown on the right. B: SerpinA1 mRNA levels were determined using quantitative real-time PCR in an immortalized nontumorigenic cell line (HaCaT) derived from NHEKs and in three Ha-ras-transformed HaCaT cell lines (A5, II4, and RT3). A5 is a benign tumorigenic HaCaT cell line, II4 forms invasive malignant tumors, and RT3 cells form metastatic SCCs in vivo. The mRNA levels were corrected for glyceraldehyde-3-phosphate dehydrogenase $($ GAPDH $)$ mRNA levels in the same samples $(n=4)$. Data are given as mean $\pm \mathrm{SD}$. Statistical difference was determined by Student's $t$-test.

\section{Expression of SerpinA1 by Cutaneous SCC Cells is Enhanced by EGF, TNF- $\alpha$, IFN- $\gamma$, and IL-1 $\beta$}

The histologic features of cutaneous SCC are characterized by infiltration of tumor microenvironment by inflammatory cells in the peritumoral area. To elucidate the regulation of SerpinA1 expression, we treated primary cutaneous SCC cells in culture for 24 hours with growth factors and cytokines (EGF, TNF- $\alpha$, IFN- $\gamma$, and IL-1 $\beta$ ) present in the tumor microenvironment of cutaneous SCCs. The mean expression level of SerpinA1 mRNA was markedly up-regulated in EGF-, TNF- $\alpha-$, IFN- $\gamma-$, and IL- $1 \beta$-treated cultures compared with untreated control cells, with IFN- $\gamma$ showing the most potent effect (Figure 3A). The conditioned media of the corresponding cultures were also analyzed by Western blotting for the production of SerpinA1. Treatment of SCC cells with EGF, TNF- $\alpha$, IFN- $\gamma$, or IL- $1 \beta$ resulted in potent enhancement of SerpinA1 production compared with untreated control cultures (Figure 3B).

\section{SerpinA1 Expression by Cutaneous SCC Cells Is Regulated by p38 MAPK}

To further investigate the regulation of SerpinA1 expression in cutaneous SCC cells, we treated primary cutaneous SCC cells with the inhibitor of MEK $1 / 2$ (PD98059) or the inhibitor of $\mathrm{p} 38 \alpha$ and $\mathrm{p} 38 \beta$ MAPKs (SB203580) for 24 hours. Basal SerpinA1 expression by the cutaneous SCC cells was potently down-regulated by SB203580 compared with the untreated control cells (Figure $3 \mathrm{C}$ ). In contrast, in parallel cultures treated with PD98059, production of SerpinA1 was not markedly different from that in untreated controls, although a potent reduction in ERK $1 / 2$ activation was noted as a marker for the effect of PD98059 (Figure 3C). These results implicate p38 $\alpha$ MAPK in the regulation of SerpinA1 expression in cutaneous SCC cells because p38 $\beta$ is not expressed in cutaneous SCC cells. ${ }^{20}$

\section{Expression of SerpinA1 by Tumor Cells in Human Cutaneous SCCs in Vivo Correlates with Tumor Progression}

The results presented previously herein show that the expression of SerpinA1 is specifically up-regulated by tumor cells derived from cutaneous SCCs. To investigate the role of SerpinA1 in progression of skin SCCs in vivo, we first analyzed its expression in TMAs consisting of UV-induced premalignant lesions of skin (actinic keratoses) $(n=36)$, in situ SCCs of skin (Bowen's disease) $(n=29)$, and UVinduced sporadic cutaneous SCCs $(n=71)$ by IHC analysis. Positive immunostaining for SerpinA1 was noted in all human premalignant and malignant tissue types studied (actinic keratosis, Bowen's disease, and sporadic SCC), but the staining intensities varied between the tissue types, and the semiquantitative analysis revealed substantial upregulation of SerpinA1, especially in invasive sporadic SCCs (Figure 4 and Table 1).

The weakest SerpinA1 staining was observed in premalignant epithelial cells in actinic keratosis; in 33 of 36 cases (92\%), SerpinA1 staining was negative (-) or weak (+) (Figure 4A and Table 1). Here, the immunostaining for SerpinA1 was analyzed in the actual premalignant cells, whereas stromal staining for SerpinA1 in the dermal layer was ignored because this staining mainly represents serum-derived AAT produced in the liver (Figure 4, A and B). In the tumor cells of Bowen's disease samples, SerpinA1 staining was either negative or weak in 26 of 29 cases (90\%) (Figure 4B). A trend toward stronger SerpinA1 staining in Bowen's disease compared with actinic keratosis was noted because only $24 \%$ of Bowen's disease samples were negative for SerpinA1 whereas $47 \%$ of actinic keratoses were negative. However, there was no statistically significant difference between actinic keratoses and Bowen's disease when all staining intensities were taken into account $(P>0.05)$ (Table 1). In sporadic 
A

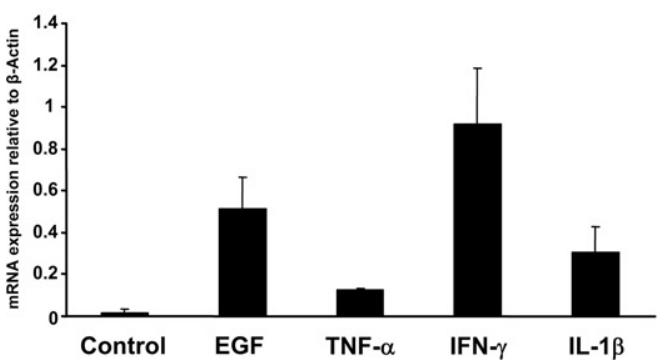

B

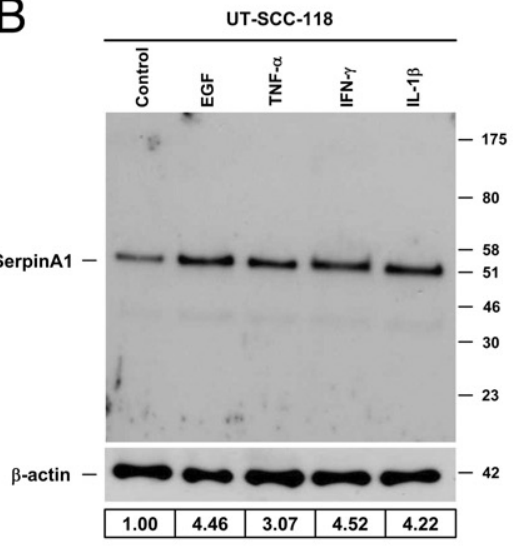

C

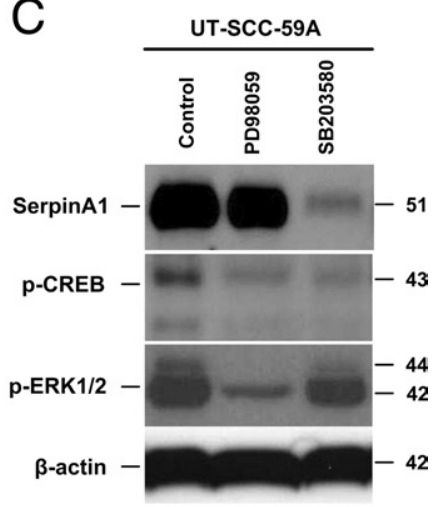

Figure 3. Up-regulation of SerpinA1 expression in cutaneous SCC cells by EGF, TNF- $\alpha$, IFN- $\gamma$, IL- $1 \beta$, and p38 signaling pathway. A: Cutaneous SCC cells (UT-SCC-59A) in culture were treated with EGF $(25 \mathrm{ng} / \mathrm{mL})$, TNF- $\alpha(20 \mathrm{ng} / \mathrm{mL})$, IFN- $\gamma(100 \mathrm{IU} / \mathrm{mL})$, and IL- $1 \beta$ (10 ng/mL) for $24 \mathrm{hours}$. The expression levels of SerpinA1 mRNA were determined using quantitative RT-PCR, and the levels were corrected for $\beta$-actin mRNA in the same samples $(n=2)$. Data are given as mean \pm SD. B: The conditioned media of the cultures in $\mathbf{A}$ were collected, and SerpinA1 levels were determined by Western blot analysis. The level of $\beta$-actin was determined in the corresponding cell lysates as an indicator of equal loading. The mean of SerpinA1 levels from three separate experiments are shown below the Western blots relative to levels in untreated control cells (1.00). Migration positions of molecular weight markers (in kilodaltons) are shown on the right. C: Cutaneous SCC cells (UT-SCC-59A) in culture were treated with MEK1/2 inhibitor PD98059 (30 $\mu$ mol/L) and p38 inhibitor SB203580 (10 $\mu$ mol/L) for 24 hours. The conditioned media were analyzed for SerpinA1 by Western blot analysis. Cell lysates were analyzed for the levels of phosphorylated CREB (p-CREB) and phosphorylated ERK1/2 (p-ERK1/2) to indicate the appropriate function of SB203580 and PD98059, respectively. The levels of $\beta$-actin were determined as a marker of the equal protein level.

cutaneous SCCs, moderate $(++)$ or strong $(+++)$ cytoplasmic staining for SerpinA1 was noted in the tumor cells in 33 of 71 cases (46\%) (Figure 4, C and E). SerpinA1 staining was significantly more abundant in invasive sporadic SCCs than in in situ Bowen's disease and actinic keratosis $(P=0.001)$ (Table 1$)$.

SerpinA1 expression was also examined in RDEBassociated SCCs, a subset of cutaneous SCCs char- acterized by aggressive behavior. The most abundant SerpinA1 expression of all SCCs examined was noted in RDEB-associated SCCs because there was clear or strong cytoplasmic staining in all 12 cases (100\%) studied (Figure 4D). Moreover, up-regulation of SerpinA1 expression was significantly more abundant in RDEB-associated SCCs than in sporadic SCCs $(P=$ 0.002) (Table 1).
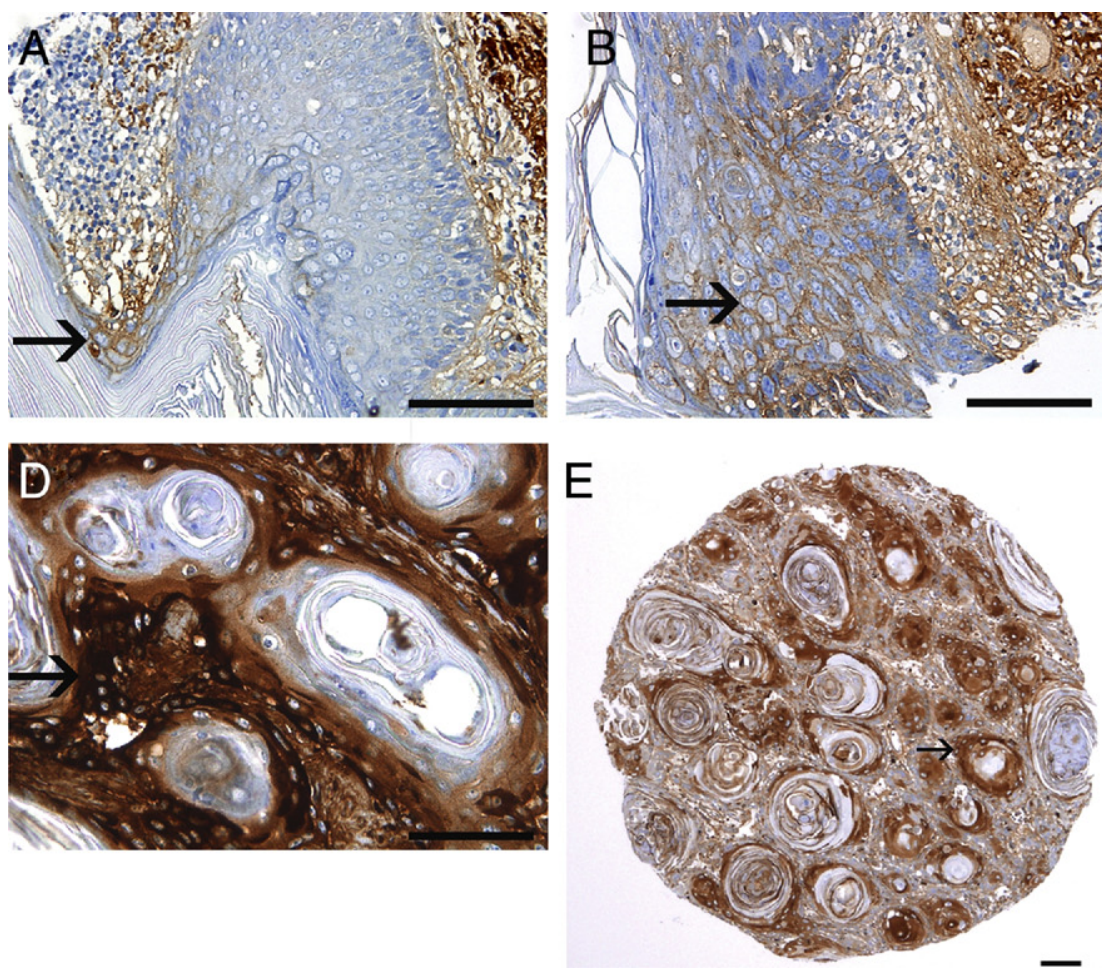

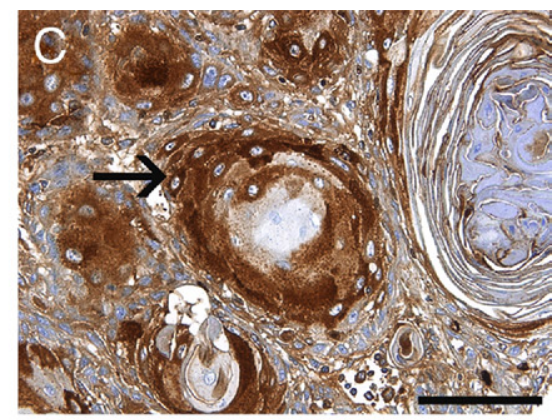

Figure 4. Expression of SerpinA1 by tumor cells in human cutaneous SCCs in vivo correlates with tumor progression. SerpinA1 expression was determined by IHC analysis in sections of TMAs consisting on UVinduced premalignant lesions (actinic keratoses), in situ SCCs (Bowen's disease), sporadic cutaneous SCCs, and RDEB-associated cutaneous SCCs, which represent clinically early metastasizing and aggressive forms of skin SCC. A: In actinic keratoses, SerpinA1 staining is typically either absent or weak (arrow). B: In Bowen's disease, weak membranous SerpinA1 immunostaining is noted (arrow). C: In sporadic UV-induced human cutaneous SCCs, clear cytoplasmic SerpinA1 staining is noted (arrow). D: Strongest SerpinA1 staining is detected in RDEB-associated cutaneous SCCs (arrow). E: Overview of the TMA sample of sporadic UV-induced human cutaneous SCCs shows clear cytoplasmic SerpinA1 staining in the tumor cells (arrow). Scale bars = $100 \mu \mathrm{m}$. 
1116 Farshchian et al

AJP September 2011, Vol. 179, No. 3

Table 1. Increased Staining Intensity for SerpinA 1 in Human Cutaneous SCCs

\begin{tabular}{lrrrr}
\hline & \multicolumn{4}{c}{ SerpinA1 staining intensity } \\
\cline { 2 - 5 } Tissue sample & \multicolumn{1}{c}{} & + & ++ & +++ \\
\hline Actinic keratosis $(n=36)$ & 17 & 16 & 3 & 0 \\
Bowen's disease $(n=29)$ & 7 & 19 & 3 & 0 \\
Sporadic SCCs $(n=71)$ & 4 & 34 & 26 & 7 \\
RDEB-associated SCCs & 0 & 0 & 3 & 9
\end{tabular}

$$
(n=12)
$$

Number of tissue specimens staining positive for SerpinA1 by $\mathrm{IHC}$ analysis in UV-induced premalignant lesions (actinic keratosis), in situ SCC (Bowen's disease), UV-induced sporadic SCCs, and RDEB-associated SCCs. Intensity of immunostaining is scored as follows: negative (-) weak $(+)$, moderate $(++)$, and strong $(+++)$. SerpinA1 staining intensity is significantly more abundant in sporadic SCCs than in Bowen's disease and actinic keratosis $\left(P=0.001\right.$, Pearson $\chi^{2}$ test) and in RDEB-associated SCCs than in sporadic SCCs $\left(P=0.002\right.$, Pearson $\chi^{2}$ test).
Table 2. Increased Staining Intensity for SerpinA1 in Chemically Induced SCCs in Mouse Skin

\begin{tabular}{ccccc}
\hline & \multicolumn{3}{c}{ SerpinA1 staining intensity } \\
\cline { 2 - 5 } Tissue sample & - & + & ++ & +++ \\
\hline $\begin{array}{c}\text { Untreated mouse skin } \\
(n=5)\end{array}$ & 5 & 0 & 0 & 0 \\
$\begin{array}{c}\text { Acetone-treated } \\
\text { mouse skin }(n=2)\end{array}$ & 2 & 0 & 0 & 0 \\
$\begin{array}{c}\text { TPA-treated mouse } \\
\text { skin }(n=6)\end{array}$ & 1 & 3 & 2 & 0 \\
Mouse SCC $(n=17)$ & 0 & 6 & 9 & 2 \\
\hline
\end{tabular}

Number of tissue specimens staining positive for SerpinA1 by IHC analysis in untreated mouse skin, vehicle (acetone)-treated skin, TPA-treated hyperplastic skin, and SCCs. Intensity of immunostaining is scored as follows: negative $(-)$, weak $(+)$, moderate $(++)$, and strong $(+++)$. SerpinA1 staining intensity is significantly more abundant in mouse SCC than in nonmalignant skin samples $\left(P=0.002\right.$, Pearson $\chi^{2}$ test $)$

acterized mouse model of chemically induced skin carcinogenesis. ${ }^{26}$ Immunostaining of the normal mouse skin, vehicle (acetone)-treated mouse skin, hyperplastic mouse skin, and chemically induced cutaneous mouse SCC was performed using specific anti-human SerpinA1 progression of cutaneous SCCs, we used the well-char-
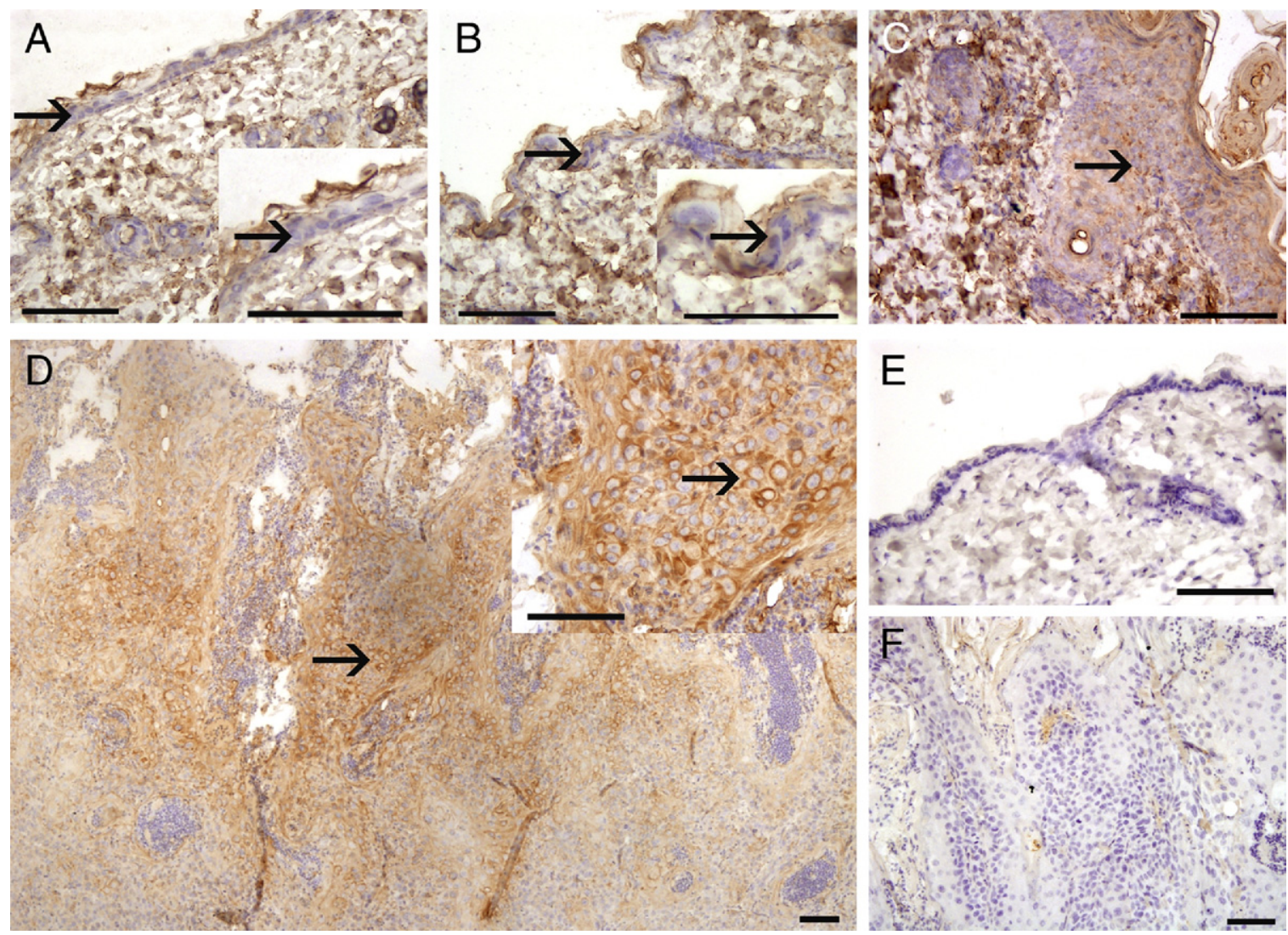

Figure 5. SerpinA1 expression correlates with progression of chemically induced mouse skin SCCs. A: In untreated control mouse skin, epidermal keratinocytes do not express SerpinA1 (arrow), whereas nonspecific staining is noted in the acellular superficial epidermal layer (stratum corneum). Higher magnification of the same area is shown in the inset. B: Treatment with vehicle (acetone) did not cause any histologic changes in mouse skin and did not induce SerpinA expression in epidermal keratinocytes (arrow). Nonspecific staining in the stratum corneum is detected. Higher magnification of the same area is shown in the inset. C: Treatment of mouse skin with TPA four times induced hyperplasia of the epidermis, and, typically, weak staining of keratinocytes for SerpinA1 was detected (arrow). D: Significant up-regulation of SerpinA1 expression was noted in DMBA-TPA-induced mouse skin SCC compared with the preceding nonmalignant samples. Clear cytoplasmic staining for SerpinA1 was noted in the mouse SCC tumor cells (arrow). Overview of the whole section shows that immunopositive cells are distributed mainly throughout the tumor. Higher magnification of the same area is shown in the inset. E: Negative control staining of untreated mouse skin. F: Negative control staining of DMBA-TPA-induced mouse skin SCC. Scale bars $=100 \mu \mathrm{m}$. 


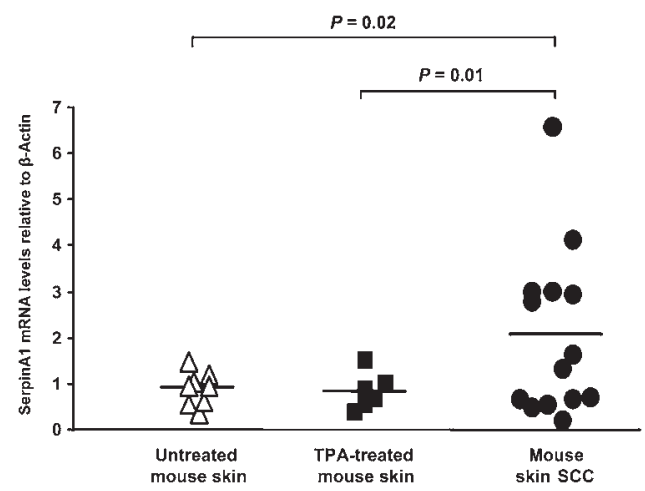

Figure 6. Overexpression of SerpinA1 in chemically induced mouse skin SCCs. SerpinA1 mRNA levels in RNAs from untreated control mouse skin $(n=8)$, TPA-treated hyperplastic skin $(n=6)$, and DMBA-TPA-induced mouse skin SCC $(n=14)$ were quantified by real-time PCR, and the levels were corrected for murine $\beta$-actin mRNA levels in the same samples. Statistical significance was determined by Student's $t$-test. Horizontal bars represent the mean SerpinA1 expression level for each group. Mean \pm SD values: control, $0.93 \pm 0.36$; TPA, $0.84 \pm 0.39$; and SCC, $2.08 \pm 1.81$.

antibody, which cross-reacts with murine SerpinA1. No SerpinA1 expression was noted in epidermal keratinocytes in normal untreated control skin or in vehicletreated skin ( $n=5$; Figure 5, A and B, and Table 2). Focal immunostaining detected in the acellular superficial epidermal layer (ie, stratum corneum) was considered nonspecific (Figure 5A). As a positive internal control, SerpinA1 immunostaining was noted in the dermal layer of mouse skin, representing serum-derived AAT (Figure 5, $A-C)$. The dermal layer in the negative-control stained region remained blank (Figure 5E). In TPA-treated mouse skin characterized by epidermal hyperplasia $(n=6)$, weak staining for SerpinA1 was noted in the keratinocytes (Figure 5C and Table 2). Prominent staining for SerpinA1 was detected in tumor cells in all mouse cutaneous SCCs studied, although the staining intensity varied from weak to strong ( $n=17$; Figure 5D and Table 2). The SerpinA1 immunostaining was distributed mainly in the center of the tumor in 8 of 17 cases $(47 \%)$ and throughout the tumor in 9 of 17 cases (53\%). Staining for SerpinA1 in SCC tumor cells was significantly stronger compared with that in all nonmalignant mouse skin samples as a group $(P=0.002)$. These results provide evidence of a similarly important role for SerpinA1 as a biomarker in progression of human and murine cutaneous SCCs.

To verify the $\mathrm{IHC}$ analysis findings, RNA samples derived from DMBA-TPA-treated mouse skin SCC $(n=14)$, hyperplastic TPA-treated skin $(n=6)$, and untreated control skin $(n=8)$ as control were analyzed using quantitative RT-PCR for SerpinA1 mRNA expression. Accordingly, the mean expression level of the SerpinA1 mRNA was significantly higher in chemically induced mouse skin SCCs than in normal or phorbol ester-treated hyperplastic skin (Figure 6). No significant difference was detected between control and TPA-treated skin.

\section{Discussion}

Serpins constitute the largest and most broadly distributed superfamily of protease inhibitors described in hu- mans. Members of this family, such as AAT, ACT, C1 inhibitor, and antithrombin, play a pivotal role in coagulation, inflammation, and turnover of extracellular matrix. $^{7,8}$ Among the members of the serpin family, SerpinA1 and SerpinA3 have been reported to be overexpressed in various malignant tumors. High expression of the SerpinA1 gene has been found in papillary thyroid cancer, ${ }^{29,30}$ lung and prostate cancer, ${ }^{14,31}$ and colorectal adenocarcinoma. ${ }^{15}$ In a recent study, SerpinA1 was among 129 genes whose elevated expression was associated with progression of esophageal squamous dysplasia. ${ }^{32}$ Elevated levels of AAT have been detected in the serum of patients with SCC of the oral cavity. ${ }^{33}$ Furthermore, expression of SerpinA3 has been documented in lung adenocarcinoma ${ }^{34}$ and in tandem with SerpinA 1 in human leukocyte antigen-positive cervical carcinoma. ${ }^{35}$

In this study, in an effort to discover novel biomarkers for the progression of cutaneous SCC, we analyzed expression of the entire serpin family in skin SCC cells using an oligonucleotide-based microarray technique. These results showed that SerpinA1 is markedly overexpressed in primary and metastatic cutaneous SCC cell lines compared with NHEKs. The overexpression of SerpinA1 mRNA was verified by quantitative real-time PCR analysis of RNAs derived from cutaneous SCC cell lines. The enhanced production of SerpinA1 protein by cutaneous SCC cells in culture was detected by Western immunoblotting. In addition, IHC analysis of TMAs consisting of a large panel (altogether 148) FFPE tissue samples from premalignant and malignant cutaneous skin tumors confirmed the findings on tumor cell-associated expression of SerpinA1 in cutaneous SCCs. Moreover, staining for SerpinA1 was weak or absent in premalignant lesions. Also, the IHC analysis using TMAs consisting of RDEBassociated cutaneous SCC tumors revealed strong tumor cell-associated SerpinA1 staining. The RDEB-associated SCC represents one of the most aggressive types of SCC, which makes the strong SerpinA1 staining in this subset of skin SCC particularly interesting. Based on these observations, we propose SerpinA1 as a novel biologic marker for the progression of cutaneous SCC.

We undertook two different experimental approaches to assess SerpinA1 as a putative marker for cutaneous carcinogenesis. First, we used an in vitro model of epidermal carcinogenesis and noted that the level of SerpinA1 expression increases, progressing from the epidermal keratinocyte-derived cell lines from immortalized nontumorigenic keratinocytes (HaCaT) (lower) toward Ha-ras-transformed $\mathrm{HaCaT}$ cell lines (higher). In addition, the level of SerpinA1 expression in Ha-ras-transformed $\mathrm{HaCaT}$ cell lines correlated with their tumorigenic potential, being lowest in the benign tumorigenic cell line (A5) and highest in RT3 cells, which form metastatic SCCs in vivo. ${ }^{23,24}$ These results also show that inactivation of p53 tumor suppressor, an early event in UV-induced epidermal carcinogenesis, is not sufficient to induce SerpinA1 expression in HaCaT cells, whereas additional activation of ras signaling pathway enhances basal SerpinA1 expression. Second, examination of DMBA-TPA-induced mouse skin SCCs, which harbor an activating $\mathrm{H}$-ras 1 mutation in addition to a p53 mutation, ${ }^{36}$ 
revealed prominent SerpinA1 expression in invasive SCCs and low expression in normal skin and hyperplastic skin lesions. The results of these two experiments imply that expression of SerpinA1 correlates with the malignant transformation of epidermal keratinocytes and provide further evidence of SerpinA1 as a biomarker for cutaneous SCC progression.

Infiltration of the tumor microenvironment with inflammatory cells is a typical histologic feature of cutaneous SCCs. ${ }^{1-3}$ Cytokines released by the inflammatory cells may promote SCC invasion and progression by stimulating the production of invasion-associated proteinases by tumor cells. Moreover, production of certain cytokines by tumor cells has been shown to regulate the local immune response against tumor cells. ${ }^{37,38}$ In the present study, we noted that the expression of SerpinA1 is enhanced by EGF and three distinct cytokines: TNF- $\alpha$, IFN- $\gamma$, and IL$1 \beta$. In addition, we noted that the basal expression of SerpinA1 in SCC cells is regulated by the p38 MAPK signaling pathway. These findings are interesting with respect to previous studies showing the role of p38 MAPKs in growth and invasion of cutaneous SCCs. ${ }^{20,39}$

Although the mechanistic role of SerpinA1 in tumorigenesis is not fully understood, previous studies have identified certain tumorigenic properties for this protein. An antiapoptotic effect of AAT on lung endothelial cells via the inhibition of caspase-3 activity has been documented. ${ }^{40}$ In addition, AAT has been shown to inhibit natural killer cell activity. ${ }^{41,42}$ Another possible tumorigenic mechanism is the mitogenic action of $\mathrm{C}$-terminal 26-residue peptide of SerpinA1, which has been shown to stimulate malignant cell proliferation with no significant effect on normal epithelial cells. ${ }^{42,43}$

Although primary cutaneous SCCs can usually be effectively treated by surgical excision, they harbor the potential for recurrence and metastasis, with the high possibility of morbidity and mortality. ${ }^{1-3}$ Because early diagnosis and excision of SCC is highly curable, prompt detection and screening are potentially lifesaving for patients. Particularly, among patients with a history and at high risk for skin cancer, regular screening is necessary to monitor the recurrence or persistence of the tumors. ${ }^{1-4}$ At present, only a few specific biomarkers for cutaneous SCC have been identified, including STAT3, ${ }^{44}$ E-cadherin, ${ }^{45}$ and matrix metalloproteinases $13,{ }^{46} 12,47$ and $7 .^{6,48}$ SerpinA1 has been previously suggested as a biomarker for the progression of insulinoma ${ }^{49}$ and thyroid cancer. ${ }^{50}$ In this study, we introduced SerpinA1 as a potential biomarker for the progression of cutaneous SCCs combined with other biomarkers mentioned previously herein.

In conclusion, the present findings show that the expression of SerpinA1 (AAT) is specifically up-regulated by cutaneous SCC cells in culture and in vivo. We also present evidence that the expression of SerpinA1 correlates with the malignant transformation of epidermal keratinocytes and their progression to invasive SCC. These results suggest that SerpinA1 could serve as a novel biomarker for the diagnosis of rapidly progressing cutaneous SCCs.

\section{Acknowledgments}

We thank Sari Pitkänen, Johanna Markola-Wärn, Sinikka Kollanus, and Sini Reponen for their skillful technical assistance; and Drs. John A. McGrath, Johann W. Bauer, Radana Königová, Márta Medvecz, Wolfgang Beckert, and Kathrin Sinemus for providing RDEB-associated SCC tissue samples.

\section{References}

1. Madan V, Lear JT, Szeimies RM: Non-melanoma skin cancer. Lancet 2010, 375:673-685

2. Rogers HW, Weinstock MA, Harris AR, Hinckley MR, Feldman SR, Fleischer AB, Coldiron BM: Incidence estimate of nonmelanoma skin cancer in the United States, 2006. Arch Dermatol 2010, 146:283-287

3. Alam M, Ratner D: Cutaneous squamous-cell carcinoma. N Engl J Med 2001, 344:975-983

4. Neville JA, Welch E, Leffell DJ: Management of nonmelanoma skin cancer in 2007. Nat Clin Pract Oncol 2007, 4:462-469

5. McGrath JA, Schofield OM, Mayou BJ, McKee PH, Eady RA: Epidermolysis bullosa complicated by squamous cell carcinoma: report of 10 cases. J Cutan Pathol 1992, 19:116-123

6. Kivisaari AK, Kallajoki M, Mirtti T, McGrath JA, Bauer JW, Weber F Konigova R, Sawamura D, Sato-Matsumura KC, Shimizu H, Csikos M, Sinemus K, Beckert W, Kähäri VM: Transformation-specific matrix metalloproteinases (MMP)-7 and MMP-13 are expressed by tumour cells in epidermolysis bullosa-associated squamous cell carcinomas. Br J Dermatol 2008, 158:778-785

7. Silverman GA, Bird PI, Carrell RW, Church FC, Coughlin PB, Gettins PG, Irving JA, Lomas DA, Luke CJ, Moyer RW, Pemberton PA, Remold-O'Donnell E, Salvesen GS, Travis J, Whisstock JC: The serpins are an expanding superfamily of structurally similar but functionally diverse proteins: evolution, mechanism of inhibition, novel functions, and a revised nomenclature. J Biol Chem 2001, 276:33293-33296

8. Law RH, Zhang Q, McGowan S, Buckle AM, Silverman GA, Wong W, Rosado CJ, Langendorf CG, Pike RN, Bird PI, Whisstock JC: An overview of the serpin superfamily. Genome Biol 2006, 7:216

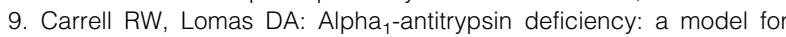
conformational diseases. N Engl J Med 2002, 346:45-53

10. Abraham CR, Selkoe DJ, Potter $\mathrm{H}$ : Immunochemical identification of the serine protease inhibitor $\alpha_{1}$-antichymotrypsin in the brain amyloid deposits of Alzheimer's disease. Cell 1988, 52:487-501

11. Kamboh MI, Minster RL, Kenney M, Ozturk A, Desai PP, Kammerer CM, DeKosky ST: Alpha-1-antichymotrypsin (ACT or SERPINA3) polymorphism may affect age-at-onset and disease duration of Alzheimer's disease. Neurobiol Aging 2006, 27:1435-1439

12. Yoon D, Kueppers F, Genta RM, Klintmalm GB, Khaoustov VI, Yoffe B: Role of alpha-1-antichymotrypsin deficiency in promoting cirrhosis in two siblings with heterozygous alpha-1-antitrypsin deficiency phenotype SZ. Gut 2002, 50:730-732

13. Higashiyama M, Doi O, Kodama K, Yokouchi H, Tateishi R: An evaluation of the prognostic significance of alpha-1-antitrypsin expression in adenocarcinomas of the lung: an immunohistochemical analysis. Br J Cancer 1992, 65:300-302

14. Karashima S, Kataoka $\mathrm{H}$, Itoh $\mathrm{H}$, Maruyama R, Koono M: Prognostic significance of alpha-1-antitrypsin in early stage of colorectal carcinomas. Int J Cancer 1990, 45:244-250

15. Tahara E, Ito H, Taniyama K, Yokozaki H, Hata J: Alpha 1-antitrypsin, alpha 1-antichymotrypsin, and alpha 2-macroglobulin in human gastric carcinomas: a retrospective immunohistochemical study. Hum Pathol 1984, 15:957-964

16. Allgayer H, Babic R, Grutzner KU, Beyer BC, Tarabichi A, Schildberg FW, Heiss MM: Tumor-associated proteases and inhibitors in gastric cancer: analysis of prognostic impact and individual risk protease patterns. Clin Exp Metastasis 1998, 16:62-73

17. Wang $Y$, Jiang $H$, Dai $D$, Su $M$, Martinka $M$, Brasher $P$, Zhang $Y$, McLean D, Zhang J, Ip W, Li G, Zhang X, Zhou Y: Alpha 1 antichymotrypsin is aberrantly expressed during melanoma progression and predicts poor survival for patients with metastatic melanoma. Pigment Cell Melanoma Res 2010, 23:575-578 
18. Chomette G, Auriol M, Vaillant JM, Kasai T, Niwa M, Mori M: An immunohistochemical study of the distribution of lysozyme, lactoferrin, alpha 1-antitrypsin and alpha 1-antichymotrypsin in salivary adenoid cystic carcinoma. Pathol Res Pract 1991, 187:1001-1008

19. Lansdorf CD, Grénman R, Bier H, Somers KD, Kim SY, Whiteside TL, Clayman GL, Welkoborsky H-J, Carey T: Cancer Cell Lines. Edited by J Masters, B Palsson. Dordrecht, Holland, Kluwer Academic Press, 1999, pp 185-255

20. Junttila MR, Ala-Aho R, Jokilehto T, Peltonen J, Kallajoki M, Grenman $\mathrm{R}$, Jaakkola $\mathrm{P}$, Westermarck J, Kähäri VM: $\mathrm{p} 38 \alpha$ and $\mathrm{p} 38 \delta$ mitogenactivated protein kinase isoforms regulate invasion and growth of head and neck squamous carcinoma cells. Oncogene 2007, 26: 5267-5279

21. Stokes A, Joutsa J, Ala-aho R, Pitchers M, Pennington CJ, Martin C Premachandra DJ, Okada Y, Peltonen J, Grénman R, James HA, Edwards DR, Kähäri VM: Expression profiles and clinical correlations of degradome components in the tumor microenvironment of head and neck squamous cell carcinoma. Clin Cancer Res 2010, 16:20222035

22. Boukamp P, Petrussevska RT, Breitkreutz D, Hornung J, Markham A, Fusenig NE: Normal keratinization in a spontaneously immortalized aneuploid human keratinocyte cell line. J Cell Biol 1988, 106:761-771

23. Boukamp P, Stanbridge EJ, Foo DY, Cerutti PA, Fusenig NE: C-Ha-ras oncogene expression in immortalized human keratinocytes (HaCaT) alters growth potential in vivo but lacks correlation with malignancy. Cancer Res 1990, 50:2840-2847

24. Mueller MM, Peter W, Mappes M, Huelsen A, Steinbauer H, Boukamp P, Vaccariello M, Garlick J, Fusenig NE: Tumor progression of skin carcinoma cells in vivo promoted by clonal selection, mutagenesis, and autocrine growth regulation by granulocyte colony-stimulating factor and granulocyte-macrophage colony-stimulating factor. Am J Pathol 2001, 159:1567-1579

25. Kivisaari AK, Kallajoki M, Ala-aho R, McGrath JA, Bauer JW Königová R, Medvecz M, Beckert W, Grénman R, Kähäri VM: Matrix metalloproteinase-7 activates heparin-binding epidermal growth factor-like growth factor in cutaneous squamous cell carcinoma. Br J Dermatol 2010, 163:726-735

26. Brideau G, Mäkinen MJ, Elamaa H, Tu H, Nilsson G, Alitalo K, Pihlajaniemi T, Heljasvaara R: Endostatin overexpression inhibits lymphangiogenesis and lymph node metastasis in mice. Cancer Res 2007, 67:11528-11535

27. Ala-aho R, Johansson N, Grenman R, Fusenig NE, Lopez-Otin C, Kähäri VM: Inhibition of collagenase-3 (MMP-13) expression in transformed human keratinocytes by interferon- $\gamma$ is associated with activation of extracellular signal-regulated kinase-1,2 and STAT1. Oncogene 2000, 19:248-257

28. Kononen J, Bubendorf L, Kallioniemi A, Bärlund M, Schraml P, Leighton S, Torhorst J, Mihatsch MJ, Sauter G, Kallioniemi OP: Tissue microarrays for high-throughput molecular profiling of tumor specimens. Nat Med 1998, 4:844-847

29. Poblete MT, Nualart F, del Pozo M, Perez JA, Figueroa CD: Alpha 1-antitrypsin expression in human thyroid papillary carcinoma. Am J Surg Pathol 1996, 20:956-963

30. Jarzab B, Wiench M, Fujarewicz K, Simek K, Jarzab M, OczkoWojciechowska M, Wloch J, Czarniecka A, Chmielik E, Lange D, Pawlaczek A, Szpak S, Gubala E, Swierniak A: Gene expression profile of papillary thyroid cancer: sources of variability and diagnostic implications. Cancer Res 2005, 65:1587-1597

31. El-Akawi ZJ, Al-Hindawi FK, Bashir NA: Alpha-1 antitrypsin (alpha1AT) plasma levels in lung, prostate and breast cancer patients. Neuro Endocrinol Lett 2008, 29:482-484

32. Joshi N, Johnson LL, Wei WQ, Abnet CC, Dong ZW, Taylor PR, Limburg PJ, Dawsey SM, Hawk ET, Qiao YL, Kirsch IR: Gene expression differences in normal esophageal mucosa associated with regression and progression of mild and moderate squamous dysplasia in a high-risk Chinese population. Cancer Res 2006, 66:6851-6860

33. Shirasuna K, Sugiyama M, Watatani K, Morioka S, Hayashido Y: Serum alpha-1-antitrypsin in patients with malignant tumors occurring in the oral region. Int J Oral Maxillofac Surg 1987, 16:516-520
34. Higashiyama M, Doi O, Yokouchi H, Kodama K, Nakamori S, Tateishi R: Alpha-1-antichymotrypsin expression in lung adenocarcinoma and its possible association with tumor progression. Cancer 1995, 76: 1368-1376

35. Kloth JN, Gorter A, Fleuren GJ, Oosting J, Uljee S, ter Haar N, Dreef EJ, Kenter GG, Jordanova ES: Elevated expression of SerpinA1 and SerpinA3 in HLA-positive cervical carcinoma. J Pathol 2008, 215: 222-230

36. Abel EL, Angel JM, Kiguchi K, DiGiovanni J: Multi-stage chemical carcinogenesis in mouse skin: fundamentals and applications. 2009 , 4:1350-1362

37. Lee SH, Jang JJ, Lee JY, Kim SY, Park WS, Shin MS, Dong SM, Na EY, Kim KM, Kim CS, Kim SH, Yoo NJ: Fas ligand is expressed in normal skin and in some cutaneous malignancies. $\mathrm{Br} \mathrm{J}$ Dermatol 1998, 139:186-191

38. Kim J, Modlin RL, Moy RL, Dubinett SM, McHugh T, Nickoloff BJ, Uyemura K: IL-10 production in cutaneous basal and squamous cell carcinomas: a mechanism for evading the local $\mathrm{T}$ cell immune response. J Immunol 1995, 155:2240-2247

39. Johansson N, Ala-aho R, Uitto V, Grénman R, Fusenig NE, Lopez-Otin C, Kähäri VM: Expression of collagenase-3 (MMP-13) and collagenase-1 (MMP-1) by transformed keratinocytes is dependent on the activity of p38 mitogen-activated protein kinase. J Cell Sci 2000, 113:227-235

40. Petrache I, Fijalkowska I, Medler TR, Skirball J, Cruz P, Zhen L, Petrache HI, Flotte TR, Tuder RM: $\alpha-1$ Antitrypsin inhibits caspase-3 activity, preventing lung endothelial cell apoptosis. Am J Pathol 2006, 169:1155-1166

41. Laine A, Leroy A, Hachulla E, Davril M, Dessaint JP: Comparison of the effects of purified human alpha 1-antichymotrypsin and alpha 1-proteinase inhibitor on NK cytotoxicity: only alpha 1-proteinase inhibitor inhibits natural killing. Clin Chim Acta 1990, 190:163-173

42. Zelvyte I, Stevens T, Westin U, Janciauskiene S: $\alpha 1$-Antitrypsin and its C-terminal fragment attenuate effects of degranulated neutrophilconditioned medium on lung cancer HCC cells, in vitro. Cancer Cell Int 2004, 4:7

43. Congote LF, Temmel N: The C-terminal 26-residue peptide of serpin A1 stimulates proliferation of breast and liver cancer cells: role of protein kinase C and CD47. FEBS Lett 2004, 576:343-347

44. Suiqing C, Min Z, Lirong C: Overexpression of phosphorylated-STAT3 correlated with the invasion and metastasis of cutaneous squamous cell carcinoma. J Dermatol 2005, 32:354-360

45. Koseki S, Aoki T, Ansai S, Hozumi Y, Mitsuhashi Y, Kondo S: An immunohistochemical study of E-cadherin expression in human squamous cell carcinoma of the skin: relationship between decreased expression of E-cadherin in the primary lesion and regional lymph node metastasis. J Dermatol 1999, 26:416-422

46. Airola K, Johansson N, Kariniemi AL, Kähäri VM, Saarialho-Kere UK: Human collagenase-3 is expressed in malignant squamous epithelium of the skin. J Invest Dermatol 1997, 109:225-231

47. Kerkelä E, Ala-aho R, Jeskanen L, Rechardt O, Grénman R, Shapiro SD, Kähäri V-M, Saarialho-Kere U: Expression of human macrophage metalloelastase (MMP-12) by tumor cells in skin cancer. J Invest Dermatol 2000, 114:1113-1119

48. Impola U, Jeskanen L, Ravanti L, Syrjänen S, Baldursson B, Kähäri VM, Saarialho-Kere U: Expression of matrix metalloproteinase (MMP)-7 and MMP-13 and loss of MMP-19 and p16 are associated with malignant progression in chronic wounds. Br J Dermatol 2005 152:720-726

49. de Sá SV, Corrëa-Giannella ML, Machado MC, Krogh K, de Almeida $M Q$, Albergaria Pereira MA, Coelho Siqueira SA, Patzina RA, Ibuki FS, Sogayar MC, Giannella-Neto D: Serpin peptidase inhibitor clade A member 1 as a potential marker for malignancy in insulinomas. Clin Cancer Res 2007, 13:5322-5330

50. Griffith OL, Melck A, Jones SJ, Wiseman SM: Meta-analysis and meta-review of thyroid cancer gene expression profiling studies identifies important diagnostic biomarkers. J Clin Oncol 2006, 24:50435051 